\title{
Conselho federal de enfermagem no transcurso da COVID-19: comunicação digital à luz da teoria habermasiana
}

RESUMO |Objetivo: Analisar as matérias publicadas pelo Conselho Federal de Enfermagem no transcurso da pandemia COVID-19 à luz da teoria da ação comunicativa habermasiana. Método: Estudo documental qualitativo, realizado durante o período de maio a julho de 2020, utilizando-se como fonte primária as notícias relacionadas à pandemia publicadas pela autarquia. O material foi organizado através da análise de conteúdo temática e discutido com base na teoria e literatura relacionada à temática. Resultados: O conselho produziu materiais notas técnicas como instrumentos norteadores aos profissionais e aprovou resoluções e normativas. A atuação do sistema esteve focada na aquisição e distribuição de equipamentos de proteção por intermédio dos regionais, ações judiciais, criação do observatório e de um canal de apoio emocional aos profissionais. Conclusão: Priorizou-se uma comunicação com sensibilização e conhecimento aos profissionais de enfermagem, empoderando-os nos processos de trabalho no contexto pandêmico.

Palavras-chaves: Pandemias; Coronavírus; Comunicação em saúde; Enfermagem; Empoderamento.

ABSTRACT | Objective: To analyze the articles published by the Federal Nursing Council during the COVID-19 pandemic in the light of the Habermasian communicative action theory. Method: Qualitative documentary study, carried out from May to July 2020, using pandemic news published by the municipality as the primary source. The material was organized through thematic content analysis and discussed based on the theory and related literature. Results: The council produced technical note materials as instruments to guide professionals and approved resolutions and regulations. The performance of the system was focused on the acquisition and distribution of protective equipment through regional offices, lawsuits, the creation of an observatory and an emotional support channel for professionals. Conclusion: Communication with awareness and knowledge to nursing professionals was prioritized, empowering them in work processes in the pandemic context.

Keywords: Pandemics; Coronavirus; Health communication; Nursing; Empowerment.

RESUMEN | Objetivo: Analizar los artículos publicados por el Consejo Federal de Enfermería durante la pandemia de COVID-19 a la luz de la teoría de la acción comunicativa habermasiana. Método: Estudio documental cualitativo, realizado de mayo a julio de 2020, utilizando como fuente primaria las noticias sobre pandemias publicadas por el municipio. El material fue organizado a través de análisis de contenido temático y discutido con base en la teoría y literatura relacionada con el tema. Resultados: El consejo elaboró materiales de notas técnicas como instrumentos para orientar a los profesionales y aprobó resoluciones y reglamentos. La actuación del sistema se centró en la adquisición y distribución de equipos de protección a través de oficinas regionales, juicios, la creación de un observatorio y un canal de apoyo emocional a los profesionales. Conclusión: Se priorizó la comunicación con conciencia y conocimiento a los profesionales de enfermería, empoderándolos en los procesos de trabajo en el contexto pandémico.

Palabras claves: Pandemias; Coronavirus; Comunicación sanitaria; Enfermería; Empoderamiento.

Mirna Neyara A. de Sá Barreto Marinho

Enfermeira. Doutoranda do Programa de Pós-Graduação Cuidados Clínicos em Enfermagem e Saúde (PPCCLIS) - Universidade Estadual do Ceará (UECE). Fortaleza-CE, Brasil.

ORCID: 0000-0002-5853-6532

\section{Rafael Bezerra Duarte}

Enfermeiro. Mestrando do Programa de Pós-Graduação em Saúde Coletiva - PPSAC, pela Universidade Estadual do Ceará - UECE. Docente do Centro Universitário Vale do Salgado - UNIVS. Brasil.

ORCID: 0000-0002-2280-0864

\section{Ana Karoline Barros Bezerra}

Enfermeira; Mestranda do Programa de Pós-Graduação Cuidados Clínicos em
Enfermagem e Saúde (PPCCLIS) - Universidade Estadual do Ceará (UECE). Fortaleza-CE, Brasil.

ORCID: 0000-0002-8249-4053

\section{André Ribeiro de Castro Júnior}

Enfermeiro. Mestre em Cuidados clínicos em enfermagem e saúde pela UECE. Fortaleza-CE, Brasil.

ORCID: 0000-0002-3681-3607

\section{Olga Maria de Alencar}

Enfermeira. Doutoranda do Programa de Pós-Graduação em Saúde Coletiva (PPSAC) da Universidade Estadual do CearUECE. Fortaleza-CE, Brasil. ORCID: 0000-0003-2477-7503

Maria Rocineide Ferreira da Silva
Enfermeira. Doutora em Saúde Coletiva

Associação Ampla UECE-UFC. Professora Adjunto do Curso de Enfermagem da Universidade Estadual do Ceará - UECE, e da Pós-Graduação nos Programas de Saúde Coletiva, Cuidados Clínicos em Enfermagem e Saúde, Saúde Coletiva e Mestrado Profissional em Saúde da Família. Fortaleza-CE, Brasil. ORCID: 0000-0002-6086-6901

Recebido em: 13/04/2021 Aprovado em: 28/04/2021

INTRODUÇÃO

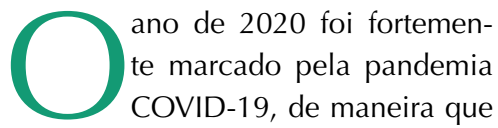
o Brasil e os demais países têm enfrenta- 
do uma crise de graves consequências à vida humana, à saúde pública e à economia. ${ }^{(1)}$ Destarte, as medidas preventivas relacionadas ao novo coronavírus, agente causador da doença, ocorreram tardiamente, considerando que apenas em 11 de março de 2020 a Organização Mundial de Saúde (OMS) a classificou como doença pandêmica. ${ }^{(2)}$

Nessa conjuntura, a COVID-19 se tornou o assunto mais comentado nos noticiários, nas conversas entre familiares, amigos e colegas de trabalho, e, também, no discurso de representantes políticos, sendo que, muitas dessas informações noticiadas/comentadas não apresentavam veracidade, comprometendo os resultados para um melhor desfecho deste cenário. ${ }^{(3)}$

A informação é uma ferramenta necessária para que as pessoas tenham conhecimento com relação à doença, suas consequências e as medidas adequadas para evitar contaminação e sua propagação. No âmbito da saúde, informação também representa poder e o seu compartilhamento em situações emergenciais como surtos, epidemias e pandemias torna-se primordial para que órgãos e serviços planejem suas ações a partir dessa comunicação. ${ }^{(4)}$

Para os profissionais de saúde os desafios são ainda maiores, pois, além de toda a demanda implicada e dificuldades vivenciadas no enfrentamento da pandemia, estes devem estar constantemente informados por meio dos diversos meios de comunicação, a fim de prestarem uma assistência qualificada e livre de danos decorrentes de negligência, imprudência ou imperícia.

Dentre esses profissionais, a categoria de enfermagem representa o maior contingente de trabalhadores nos serviços públicos e privados, fazendo-se presentes em todos os municípios brasileiros, níveis de atenção à saúde e estruturas organizacionais do sistema. ${ }^{(5)}$ Para tanto, o Conselho Federal de Enfermagem (COFEN) demonstrou preocupação com os profissionais de enfermagem nesse contexto, utilizando-se de sua página na internet para difundir in- formações diversas aos profissionais de enfermagem e à sociedade civil.

A internet se configura na atualidade como uma importante Tecnologia Digital de Informação e Comunicação, com incontáveis fontes de dados e possibilidades de interações entre as pessoas, e, no campo da saúde, há um número crescente de usuários e profissionais que buscam por informações no seu cotidiano, o que pode lhes proporcionar uma maior segurança e autonomia. ${ }^{(6)}$

Diante desses argumentos, contempla-se a Teoria da Ação Comunicativa (TAC), proposta Jürgen Habermas, pesquisador expoente da teoria crítica e considerado um dos grandes pensadores da atualidade. (7) Assim, questiona-se: Qual o enfoque comunicativo da autarquia direcionado à categoria da enfermagem no que se relaciona à pandemia? Nesse direcionamento, objetivou-se com este estudo analisar as matérias publicadas pelo COFEN no transcurso da pandemia COVID-19 à luz da TAC.

\section{MÉTODO}

Trata-se de estudo documental de abordagem qualitativa, com coleta de dados realizada durante os meses de maio a julho de 2020, através da página do COFEN (www.cofen.gov.br), utilizando-se das matérias relacionadas à pandemia $\mathrm{CO}$ VID-19 desde a primeira, publicada em 14.02.2020 até a última do mês de junho de 2020, totalizando 280.

O material empírico foi analisado através da análise de conteúdo temática e discutido com base na TAC e literatura relacionada ao novo coronavírus. ${ }^{(8)} \mathrm{A}$ base para a constituição do corpus do material se deu por meio das notícias que foram produzidas diretamente pela Assessoria de Comunicação (ASCOM) do COFEN. No entanto, faz-se importante destacar que todas as notícias foram lidas para classificação e selecionadas as que pudessem ser discutidas com a teoria.

Para a intepretação dos resultados, tomou-se como base a TAC, que considera o 'agir comunicativo' como uma inclusão de situações de fala, da aplicação da linguagem, dos contextos, das pretensões e dos papéis do diálogo. A ação comunicativa contribui para o entendimento do trabalho em equipe de saúde, por considerar que as abordagens teóricas possuem como finalidades a busca de possibilidades de explicação ou compreensão de um determinado fenômeno e, sobretudo, de intervenção na realidade. ${ }^{(9)}$

Por se tratar de pesquisa com informações de domínio público, dispensa-se a necessidade de registro pela Plataforma Brasil para avaliação por Comitê de Ética em Pesquisa designado. ${ }^{(10)}$

\section{RESULTADOS}

Os trechos das matérias inseridas tinham como foco a atuação do COFEN no contexto da pandemia, a partir das orientações/esclarecimentos aos profissionais no tocante às informações sobre a doença e as ações da entidade no enfrentamento da doença. Destarte, a intepretação do material empírico proporcionou a criação das seguintes categorias temáticas: 'Comunicação em tempos de COVID-19: Orientações do COFEN aos profissionais' e 'O agir do COFEN no contexto da COVID-19: O papel da entidade neste cenário'.

Comunicação em tempos de COVID-19: orientações do COFEN aos profissionais

Para esta categoria, menciona-se que o conselho produziu notas técnicas como instrumentos norteadores aos profissionais, aprovando resoluções e normativas, orientando o processo de trabalho no enfrentamento à pandemia.

O COFEN implantou, logo após a OMS ter declarado a COVID-19 como uma pandemia, o Comitê Gestor de Crise (CGC) no âmbito do sistema, com o objetivo de gerenciamento e elaboração de recomendações e estratégias de atuação emergencial, com base nas previsões do Ministério da Saúde e demais autoridades sanitárias.(11) Assim, o CGC tornou-se o principal responsável no âmbito do sistema COFEN/COREN de acompanhar as 
normativas e a partir delas trazer orientações para a enfermagem, conforme apontam os trechos que se seguem:

O Comitê de Crise tem recebido pedidos de informação de profissionais de todo o Brasil (...). "Preparamos um documento orientador, em consonância com as recomendações da Organização Mundial de Saúde, Ministério da Saúde (MS e demais autoridades sanitárias)". (Notícia - 20/03/2020).

O Cofen publicou hoje nova versão das diretrizes para a organização dos serviços de saúde frente à pandemia de COVID-19. As atualizações incluem mudança nas indicações de usos de equipamentos de proteção individual, considerando a mudança na orientação da OMS e o avanço da doença no Brasil. (Notícia - 23/04/2020).

No que se refere à legislação aprovada para este período, destaca-se a teleconsulta de enfermeiros e o dimensionamento de pessoal de enfermagem:

A Resolução 634/2020 autoriza que os enfermeiros realizem consultas, orientações e encaminhamentos por meios tecnológicos. "A participação dos enfermeiros no combate à pandemia é fundamental, principalmente nesses momentos de isolamento social, em que as pessoas precisam de acesso a informações seguras e com possibilidade de atendimento sem deslocamentos às unidades de saúde". (Notícia - 27/03/2020).

O Conselho Federal de Enfermagem publicou parecer normativo que estabelece o quantitativo mínimo da equipe de Enfermagem necessária para a adequada assistência aos pacientes acometidos pela COVID-19(...). (Notícia 18/05/2020).
A segunda categoria expressa às ações mais relevantes do COFEN no cenário da COVID-19.

O agir do COFEN no contexto da COVID-19: o papel da entidade neste cenário

A atuação do sistema esteve focada na aquisição e distribuição de EPI por intermédio dos COREN, ações formalizadas junto ao Ministério Público e o poder judiciário, criação do observatório de enfermagem e de um canal de apoio em saúde mental aos profissionais.

No que tange aos EPI, o Brasil, assim como outros países do mundo, enfrentou sérios problemas inicialmente com relação à compra e distribuição, o que gerou dificuldades na logística, além do aumento da necessidade em larga escala.

Os trechos abaixo demonstram as ações do COFEN no tocante à problemática de EPI:

Em resposta aos relatos de profissionais sobre o estado crítico das condições de trabalho e falta de EPI, o Cofen publicou hoje chamamento público para a compra de máscaras de proteção N95. (Notícia - 25/03/2020).

(...) Diante dos relatos generalizados de falta de EPIs em unidades de Saúde, o Sistema Cofen/Conselhos Regionais vem articulando soluções junto ao poder público e somando esforços para prover os equipamentos necessários. Corens de todas as regiões do Brasil têm distribuído materiais, como as máscaras cirúrgicas e as N95, indicadas em procedimentos mais invasivos. (Notícia - 02/04/2020).

Outras ações da entidade se deram no campo da judicialização. Os profissionais de enfermagem, por desempenharem cuidados aos pacientes durante às 24 horas, foram os mais susceptíveis ao adoecimento pela COVID-19 e, consequentemente, risco de morte. Assim, o COFEN travou uma luta para conseguir o afastamento na linha de frente dos profissionais com idade avançada e/ou do grupo de risco, além de ter focado a necessidade de testagem global, conforme apontam os trechos a seguir:

As diretrizes do Cofen para organização dos serviços de Saúde durante a pandemia preconizam que esses profissionais devem permanecer na retaguarda, em funções administrativas, que também são imprescindíveis. (Notícia - 17/04/2020).

O Cofen ajuizou ação cível pública, com pedido de tutela de urgência, para assegurar a realização de testes rápidos de detecção do novo coronavírus nos profissionais de Enfermagem. A testagem ampla é um dos pilares para o controle da doença. (Notícia - 24/04/2020).

Para além da criação do CGC e do observatório, que tem possibilitado ao COFEN a análise do cenário nacional para a implementação de ações, instituiu-se também o canal de apoio emocional aos profissionais envolvidos no trabalho da pandemia, diariamente, durante as 24 horas. Seguem trechos da atuação do COFEN relacionadas a esses dispositivos:

Gabinete de Crise criado pelo Cofen está acompanhando diariamente o impacto da pandemia de Covid-19 para os profissionais de Enfermagem. O objetivo é coIher informações para direcionar as ações do sistema, monitorar os dados epidemiológicos e os Equipamentos de Proteção Individual (EPIs) em cada estado, dentre outras ações. (Notícia - 30/04/2020). (...) O Cofen disponibilizará um canal de atendimento $24 \mathrm{~h}$, todos os dias da semana, para que os profissionais de Enfermagem possam procurar ajuda emocional em meio à situação de pandemia do Coronavírus (....). (Notícia - 25/03/2020). 
DISCUSSÃO

Muitas foram às demandas anunciadas pelos profissionais de enfermagem em meio à crise gerada pela COVID-19, tanto pelo canal das ouvidorias do sistema, como pelas informações geradas para o CGC a partir da fiscalização do exercício profissional. O documento elaborado pelo comitê buscava a organização dos serviços para a assistência a ser prestada mediante o novo coronavírus, com orientações que incluíam formação de equipe de resposta rápida, definição de pontos de recepção para sintomáticos respiratórios e demais medidas de adequação à assistência de enfermagem a serem observadas pelos serviços, almejando uma maior segurança e proteção aos profissionais de enfermagem. ${ }^{(11-12)}$

A comunicação imbricada para a categoria está associada, no que se refere a essas orientações, a uma melhor compreensão de posturas e práticas nessa situação de crise. Para Habermas, a comunicação é mediada pela linguagem e parte do pressuposto de que esta carrega nela mesma um telos comunicativo, para a busca de entendimento. Assim, reconhece-se que a ciência, por mais especializada que seja, necessita da moldura da linguagem comum, não apenas na fase de divulgação, mas também de sua produção. ${ }^{(9,13)}$

$A$ realidade descortinada com a $\mathrm{CO}$ VID-19 levou o COFEN a repensar algumas práticas da profissão, autorizando inclusive a modalidade de teleconsulta, possibilitando o contato do enfermeiro com o paciente mesmo à distância de forma simultânea ou assíncrona, com orientações para a utilização dos meios eletrônicos, respeitando-se os princípios éticos e legais da profissão. ${ }^{(14)}$

O dimensionamento de profissionais de enfermagem nas instituições públicas e privadas, amplamente debatida pela Resolução COFEN 543/2017, necessitou de adaptações para este cenário, considerando inclusive a criação dos hospitais de campanha em todo o país. A normativa recomendava, além de alterações de cálculo, um Índice de Segurança Téc- nica de $15 \%$ para $20 \%$, em virtude do expressivo aumento do número de profissionais afastados acometidos também pela doença. ${ }^{(15-16)}$

Na TAC, são abordados aspectos que fundamentam a compreensão do entendimento mútuo e da interface entre o mundo social e o agir pautado em normas, partindo-se do princípio que através da linguagem os homens constituem-se sujeitos de ação, estabelecendo um contexto de relações e interações sociais que desencadeiam processos de mudança. Através dos meios de comunicação, a esfera pública faz a mediação de uma estrutura comunicacional referente ao espaço social gerado na ação comunicativa e em sua capacidade de liderança. ${ }^{(17-18)}$

Para tanto, a página do COFEN na internet tem apresentado aos profissionais estas normativas, possibilitando-Ihes conhecimento e atualização para ampliação de sua capacidade de argumentação nos serviços frente às situações desafiadoras e conflitantes geradas pela COVID-19. Pela sua representatividade política e atribuições conferidas por lei, o COFEN possui o dever e a responsabilidade de utilizar os múltiplos mecanismos de comunicação para que estas informações alcancem a comunidade da enfermagem em todo o país.

Com relação às dificuldades de EPI, os serviços, seguindo as recomendações da Agência Nacional de Vigilância Sanitária, passaram a contingenciar os mais utilizados, a exemplo da máscara N95/ PFF2, recomendada para a realização de dispositivos que gerem aerossóis, com orientações para reutilização prolongada desse dispositivo. ${ }^{(19)}$ Com a compra realizada pelo COFEN, os estados brasileiros receberam estes dispositivos por intermédio dos Conselhos Regionais.

O pedido de afastamento dos profissionais de enfermagem considerados grupos de risco se deu com base nos dados gerados pelo observatório de enfermagem, plataforma criada com o propósito de monitorar os casos e óbitos relacionados à doença em todo o país, de modo que estes pudessem ser realocados em atividades de gestão, suporte ou assistência nas áreas em que não ocorresse o contato com pacientes suspeitos ou contaminados pela COVID-19. A autarquia conquistou o direito de testagem dos profissionais, independente de sintomas clínicos, desde que comprovada sua participação no enfrentamento à pandemia. ${ }^{(20-21)}$

O canal de apoio emocional foi criado com o objetivo de oferecer suporte aos profissionais de enfermagem na linha de frente à COVID-19, realizado por enfermeiros com titulações no campo da saúde mental com atendimentos via live chat após preenchimento de formulário, mantendo-se sigilo e anonimato das demandas. ${ }^{(22)}$

Os profissionais de enfermagem têm experimentado por todo o país, além do desgaste emocional/mental em consequência da pandemia, alto índice de adoecimento e morte, conforme dados apontados pelo observatório de enfermagem, colocando o Brasil no ranking do maior número de óbitos de enfermeiros em todo o mundo. Por estar nessa frente em seu trabalho é sentido o peso do desgaste físico e emocional. ${ }^{(23-24)}$ Os dados trazidos pelo observatório de enfermagem e pelo CGC demonstram que a situação exige políticas públicas eficazes em todas as instâncias governamentais, com ações que prezem por esta categoria, considerando os problemas ainda enfrentados mesmo com o advento da vacina.

Analisando essas ações do COFEN à luz da teoria habermasiana, reforça-se a linguagem como pano de fundo, fundamental aos processos comunicativos, a partir do que se compreende, sente-se e acessa-se aos significados e às temáticas que envolvem o mundo, trazendo, a partir da racionalidade imposta pela linguagem, um amplo horizonte de interação comunicativa. ${ }^{(25)}$

Vis-á-vis a esses argumentos, o COFEN tem buscado, para além de uma comunicação com enfoque na orientação e esclarecimentos sobre suas ações nesse momento, que a comunidade de enfermagem, gestores, políticos, magistrados e a população de maneira geral, perceba 
e compreenda a situação tal como ela se apresenta e que ela possa trazer benefícios para a enfermagem posteriormente.

\section{CONCLUSÃO}

O COFEN oportunizou uma comunicação com sensibilização e conhecimento aos profissionais, de maneira à empoderá-los nos processos de trabalho para que estes lutem por melhores condi- ções de trabalho e pelo cumprimento das normas sanitárias, minimizando assim o impacto da doença. Esta comunicação, além de necessária, configura-se um ato político, permitindo que a enfermagem possa dialogar a respeito de suas demandas e desafios nos diferentes cenários de trabalho com seus pares. A autarquia tem buscado dar visibilidade de como a pandemia tem modificado a vida e o trabalho da categoria, nos mais diversos contex- tos, e do quanto esta se faz necessária em todos os momentos da assistência.

A utilização de uma teoria comunicativa para investigações dessa natureza enriquece a análise e aponta para outros caminhos, inclusive, para a possibilidade de estudar a comunicação desenvolvida pelas demais entidades de classe na enfermagem, a exemplo da Associação Brasileira de Enfermagem, Federação Nacional dos Enfermeiros e sindicatos.

\section{Referências}

1. Caetano R, Silva AB, Guedes ACCM, Paiva CCN, Ribeiro GR, Santos DL, et al. Desafios e oportunidades para telessaúde em tempos da pandemia pela COVID-19: uma reflexão sobre os espaços e iniciativas no contexto brasileiro. Cad. Saúde Pública. [internet]. 2020 [cited 2021 Feb 5]; 36(5):1-16. D0I 10.1590/0102-311 x00088920

2. Jesus, RS, Santos PP, Sousa TD, Oliveira A, Avelar KES. Os desafios do governo brasileiro para o enfrentamento da pandemia do coronavírus. Rev. Augustus. [internet]. 2020 [cited 2021 Feb 7];25(51):31-55. DOI 10.15202/1981896.2020v25n51p31

3. Facchini LA. COVID-19: Nocaute do neoliberalismo? Será possível fortalecer os princípios históricos do SUS e da APS em meio à pandemia? APS em revista. [internet]. 2020 [cited 2021 Feb 5];2(1):3-10. DOI 10.14295/aps.v2i1.73 4. Sousa Júnior JH, Raasch $M$, Soares JC, Ribeiro LVHAS. Da desinformação ao caos: uma análise das fake news frente à pandemia do Coronavírus (COVID-19) no Brasil. Cadernos de Prospecção. [internet]. 2020 [cited 2021 Feb 8];13(2)331-346. DOI 10.9771/cp.v13i2.COVID-19.35978

5. Souza LP, Souza AG. Enfermagem brasileira na linha de frente contra o novo Coronavírus: quem cuidará de quem cuida? J. nurs. health. [internet]. 2020 [cited 2021 Feb 10];10(n.esp.):e20104005. Avaliable from: https://docs. bvsalud.org/biblioref/2020/05/1095606/1-enfermagem-brasileira-na-linha-de-frente-contra-o-novo-coron_ygPksqt.pdf

6. Pereira Neto AF, Paolucci R, Daumas RP, Souza RV. Avaliação participativa da qualidade da informação de saúde na internet: o caso de sites de dengue. Ciênc. saúde coletiva. [internet]. 2017 [cited 2021 Jan 23]. 22(6):1955-1968. DOI 10.1590/1413-81232017226.04412016.

7. Sena AAG. Teorias da Comunicação. $7^{\mathrm{a}}$ ed., Rio de Janeiro: SESES. 2017.

8. Minayo MCS. 0 desafio do conhecimento: pesquisa qualitativa em saúde. $13^{a}$ ed., São Paulo: Hucitec, 2013.

9. Habermas J. Teoria de la acción comunicativa: racionalidad de la acción y racionalización social. Madrid: Taurus; 2001.

10. Brasil. Ministério da Saúde. Conselho Nacional de Saúde. Resolução nº 510 , de 7 de abril de 2016.

11. Conselho Federal de Enfermagem. Portaria COFEN $n^{\circ} 251$, de 12 de março de 2020. Cria e constitui Comitê Gestor de Crise - CGC, no âmbito do Sistema Cofen/Conselhos Regionais de Enfermagem. COFEN. [internet]. 2020 [cited 2021 Jan 15]. Avaliable from: http://www.cofen.gov.br/portaria-cofen-no-251-de-12-de-marco-de-2020_77868.html

12. Conselho Federal de Enfermagem. Cofen publica diretrizes para serviços de enfermagem frente o COVID-19. COFEN, 2020. [internet]. 2020 [cited 2020 Dec 26]. Avaliable from: http://www.cofen.gov.br/cofen-publica-diretrizes-para-servicos-de-enfermagem-frente-o-covid-19_78031.html

13. Rivera FJU, Artmann E. Inovação e agir comunicativo: redes e tecnologias de gestão para a saúde. Cad. Saúde Pública. [internet]. 2016 [cited 2021 Feb 17];32(Sup-2):e00177014. DOI 10.1590/0102-311X00177014

14. Conselho Federal de Enfermagem. Resolução COFEN n 634/2020. COFEN. [internet]. 2020 [cited 2021 Feb 17]. Avaliable from: http://www.cofen.gov.br/ resolucao-cofen-no-0634-2020_78344.html
15. Conselho Federal de Enfermagem. Resolução COFEN n ${ }^{\circ}$ 543/2017. COFEN. [internet]. 2017 [cited 2021 Feb 20]. Avaliable from: http://www.cofen.gov.br/ resolucao-cofen-5432017_51440.html

16. Conselho Federal de Enfermagem. Parecer normativo $n^{\circ} 002 / 2020$. COFEN. [internet]. 2020 [cited 2021 Feb 02]. Avaliable from: http://www.cofen.gov.br/ parecer-normativo-no-002-2020_79941.html

17. Alves KYA, Bezerril MS, Salvador PTCO, Feijão AR, Santos VEP. Comunicação efetiva em enfermagem à luz de Jurgen Habermas. Rev Min Enferm. [internet]. 2018 [cited 2021 Jan 15]; 22:e-1147. DOI 10.5935/1415-2762.20180078

18. Menezes Júnior AS, Brzezinski I. A teoria do agir comunicativo e a formação médica: análise crítica das competências curriculares e da relação médico-paciente. EccoS - Rev. Cient. [internet]. 2018 [cited 2021 Jan 03];47:441-456. DOI 10.5585/EccoS.n47.8757

19. Agência Nacional de Vigilância Sanitária. Nota técnica GVIMS/GGTES/ANVISA $n^{\circ}$ 04/2020. Orientações para serviços de saúde: Medidas de prevenção e controle que devem ser adotadas durante a assistência aos casos suspeitos ou confirmados de infecção pelo novo coronavírus (Sars-CoV-2). [internet]. 2017 [cited 2021 Feb 04]. Avaliable from: https://www.gov.br/anvisa/pt-br/ centraisdeconteudo/publicacoes/servicosdesaude/notas-tecnicas/nota-tecnica-gvims_ggtes_anvisa-04_2020-25-02-para-o-site.pdf

20. Conselho Federal de Enfermagem. Vitória do Cofen garante proteção a profissionais em grupos de risco. COFEN. [internet]. 2017 [cited $2021 \mathrm{Feb} 08$ ]. Avaliable from: http://www.cofen.gov.br/vitoria-do-cofen-garante-protecao-a-profissionais-em-grupos-de-risco_79503.html

21. Conselho Federal de Enfermagem. Justiça assegura direito a testagem de profissionais de Saúde para covid-19. COFEN. [internet]. 2017 [cited $2021 \mathrm{Feb}$ 10]. Avaliable from: http://www.cofen.gov.br/justica-assegura-direito-a-testagem-de-profissionais-de-saude-para-covid-19_80202.html

22. Humerez DC, Ohl RIB, Silva MCN. Saúde mental dos profissionais de enfermagem do Brasil no contexto da pandemia COVID-19: Ação do Conselho Federal de Enfermagem. Cogitare enferm. [internet]. 2020 [cited 2021 Feb 15];25:e74115. DOI dx.doi.org/10.5380/ce.v25i0.74115

23. Persegona MFM, Pires RA, Medeiros GG, Pinheiro FAS, Lopes MSS, Nascimento Júnior, $A$. et. al. Observatório da enfermagem: Ferramenta de monitoramento da COVID-19 em profissionais da enfermagem. Enferm. Foco. [internet]. 2020 [cited 2021 Feb 02]; 11(Esp. 2): 6-11. Avaliable from: http://revista.cofen. gov.br/index.php/enfermagem/article/view/4283/976.

24. Reis LM, Lago PN, Carvalho AHS, Nobre VN, Guimarães AP. Atuação da enfermagem no cenário da pandemia COVID-19. Revista Nursing. [Internet]. 2020 [cited 2021 Apr 27];23(269):4765-4768. DOI 10.36489/nursing. 2020v23i269p4765-4772

25. Sturza JM, Rodrigues BP. Diálogos entre políticas e direito à saúde: As audiências públicas enquanto instrumento de participação popular sob a perspectiva da teoria da ação comunicativa de Habermas. Rev. Direitos Sociais e Políticas Públicas. [internet]. 2019 [cited 2021 Feb 03];7(2):375-411. Avaliable from: https://www.unifafibe.com.br/revista/index.php/direitos-sociais-politicas-pub/article/view/402 